\title{
Cytochemical studies of cell viability and gene expression in Bacillus subtilis macrofibres
}

\author{
Neil H. Mendelson, ${ }^{1}$ Bachira Salhi ${ }^{2}$ and Katherine E. Sundahl ${ }^{3} \dagger$ \\ Author for correspondence: Neil H. Mendelson. Tel: +1520621 3617. Fax: +15206213709. \\ e-mail: neil_mendelson@tikal.biosci.arizona.edu
}

Department of Molecular and Cellular Biology',

Department of

Microbiology and

Immunology ${ }^{2}$ and

Department of

Biochemistry ${ }^{3}$, University

of Arizona, PO Box

210106, Tucson, AZ 85721-

0106, USA

\begin{abstract}
The viability of cells and the expression of a reporter gene in Bacillus subtilis macrofibres have been examined using cytochemical approaches. Previous studies have shown that macrofibres grow at an exponential rate in fluid environments and undergo complex dynamic motions as they elongate but the behaviour of individual cells in them has never been examined. A fluorescence staining procedure that distinguishes living cells from dead cells was used in the experiments described to examine cells in both left- and right-handed macrofibres. Very few dead cells were found in individual fibres. Their locations appeared to be random, suggesting that neither the extreme shape deformation imposed upon cells in the loop ends of fibres, nor the entrapment of cells in the interior of a fibre compromise viability. In related experiments, initial studies of reporter gene expression during fibre morphogenesis were conducted using a strain previously studied as conventional colonies. Spatial and temporal differences in the appearance of the blue cleavage product of $X$ Gal were found, suggesting that differential gene expression may arise in macrofibres in contrast to the results found in colonies. Two conclusions may be drawn from the findings: (i) virtually all cells in macrofibres are viable and (ii) all the cells in macrofibres do not always behave in an identical manner.
\end{abstract}

Keywords: cell viability, multicellularity, differential gene expression, $B$. subtilis macrofibres

\section{INTRODUCTION}

Multicellular states always impose constraints upon the individual cells that comprise them. In bacterial colonies, for example, physical factors may affect cell motility and colony expansion, whereas chemical constraints such as nutrient gradients can govern gene expression and colony development (Salhi \& Mendelson, 1993; Ben-Jacob et al., 1994; Mendelson \& Salhi, 1996). In Bacillus subtilis macrofibres, a multicellular state in which all the cells remain attached to one another in a single filament, twisting motions that accompany cell growth cause writhing and bending, deformation of cell shape from cylindrical to helical form and supercoiling of the cellular filament (Mendelson, 1992). These dynamic processes are all caused by forces acting upon the cell wall that arise during cell growth. Because the cells are organized into a multicellular filament, however, their response to forces

† Present address : 2836 SW Sam Jackson Park Road, Portland, OR 97201, USA. differs from that of individual cells subjected to the same forces. Mechanical constraints come into play in the multicellular filament and they in turn cause macrofibres to form (Mendelson \& Thwaites, 1989).

A mature macrofibre produced in a fluid environment consists of a structure several millimetres in length. During its self-assembly, the single cell filament folds back upon itself numerous times. Following each fold, the filament twists together giving rise to a fibre-like structure with loops on both ends and a shaft in between. The fibre shaft eventually contains tens to hundreds of parallel segments of the long single cell filament. Macrofibre morphogenesis causes individual cells to reside in different locations at different times. Cells situated in the fibre shaft, for example, later may become part of a terminal loop as a result of folding, whereas cells in a terminal loop can become part of the shaft. Cells on the surface of a young fibre may eventually reside in the interior of the filament bundle because each folding increases the thickness of the fibre and the number of filament segments aligned parallel to each other. At very late stages macrofibres condense into a 
ball-like form. New cellular environments are created by this change of shape. Thus, although all cells in a macrofibre are members of a single clone and part of a single filament, each cell at any given time is subject to a unique environment.

In this study we have examined the viability of cells in macrofibres and the ability of macrofibre cells to express a reporter gene. It is important to know whether cells die in any specific regions of macrofibres to properly interpret the relationship of individual cell growth to macrofibre self-assembly. Studies of gene expression in macrofibres are directed towards understanding how multicellularity influences cell regulation. We have previously investigated similar issues using bacterial colonies (Mendelson \& Salhi, 1996). The findings described here show that individual cells in macrofibres behave differently, suggesting that they may be able to sense and respond to their location within fibres.

\section{METHODS}

Strains. Bacillus subtilis 168 strains FJ7 and F2b were used in this investigation. FJ7 carries met $\mathrm{C}$ and $l y t-2$ markers. F2b carries metC lyt-2 $\mathrm{Em}^{\mathrm{r}} \operatorname{Lin}^{\mathrm{r}}$ and $\operatorname{Tn} 917$ (lacZ). F2b is a derivative of strain FJ7 produced as described by Salhi \& Mendelson (1993). The transposon Tn917 carrying the Escherichia coli lacZ gene minus its promoter was used to introduce the reporter gene into the host chromosome at random locations by forced transposition from a suicide plasmid, PTV32ts (Youngman, 1987). Antibiotic-resistant isolates were screened for lacZ expression. Loss of the suicide plasmid was shown by chloramphenicol sensitivity and the absence of plasmid DNA on agarose gels. F2b produces conventional macrofibres when grown as described below (Mendelson, 1978).

Media and growth conditions. The complex medium TB (Mendelson, 1976) consisting of tryptose (10 g, Difco), beef extract $(3 \mathrm{~g}, \mathrm{Difco})$ and $\mathrm{NaCl}$ [ $5 \mathrm{~g}$ (1 deionized water $\left.)^{-1}\right]$ was the liquid medium used for the growth of strains. TB medium was supplemented with magnesium sulfate or ammonium sulfate to obtain either right- or left-handed fibres, respectively, as described previously (Mendelson \& Favre, 1987).

Macrofibres were produced in liquid TB media under static conditions at $20^{\circ} \mathrm{C}$ as described previously (Mendelson, 1978). They were subcultured daily by transferring small fragments of young fibres to fresh TB medium.
Cultures of FJ7 that grew in the form of webs rather than macrofibres were also produced in TB medium (Thwaites \& Mendelson, 1985). Such cultures contain long single cell filaments that can be drawn manually into structures known as bacterial threads. Samples of the web and of threads drawn from the web that were dried then re-hydrated were used as controls in the live/dead cytochemical determinations.

Strains were maintained as streaks on 33 g tryptose blood agar base (TBAB; $10 \mathrm{~g}$ tryptose, $3 \mathrm{~g}$ beef extract, $5 \mathrm{~g} \mathrm{NaCl}$ and $15 \mathrm{~g}$ agar per $33 \mathrm{~g}$ ) medium (Difco) plus $5 \mathrm{~g}$ Bacto Agar (Difco) (l deionized water) ${ }^{-1}$.

Determination of cell viability in macrofibres. The Live/Dead Bac Light Bacteria Viability Kit (L-7007, Molecular Probes, Inc.) was used to visualize live and dead cells in macrofibres. The method is based on a proprietary mixture of nucleic acid stains that distinguishes cells with intact membranes (live cells stain fluorescent green) from those with damaged membranes (dead cells stain fluorescent red). Following the supplier's instructions, equal amounts of the two dyes provided were mixed together. Six microlitres of the fresh stain mixture was added to $1 \mathrm{ml}$ TB medium and $20 \mu$ volumes of the solution were transferred to microscope slides. Macrofibres produced by overnight growth at $20^{\circ} \mathrm{C}$ in TB medium supplemented with either magnesium sulfate (for right-handed fibres) or ammonium sulfate (for left-handed fibres) (Mendelson \& Favre, 1987) were transferred with a Pasteur pipette directly into the $20 \mu \mathrm{l}$ stain/TB solution. The final volume was approximately $40 \mu \mathrm{l}$. After addition of a coverslip the slides were incubated for $10 \mathrm{~min}$ at $23^{\circ} \mathrm{C}$ in the dark before viewing.

A similar protocol was applied to samples of bacterial webs and the threads drawn from them to illustrate that the live/dead detection system was capable of resolving a few cells of either category amongst a majority of the others. The cell filaments in approximately $0.05 \mathrm{ml}$ of a bacterial web culture were added to an equal volume of the stain mixture and after a brief incubation, $10 \mu \mathrm{l}$ was transferred to a microscope slide, covered with a coverslip and incubated in the dark as described above. These specimens were used to illustrate the detection of a few dead cells in a population consisting almost entirely of viable cells. The same fresh stain mixture $(1.5 \mu \mathrm{l})$ was also added to $0.5 \mathrm{ml} \mathrm{TB}$ and $1-2 \mathrm{~mm}$ of dried bacterial thread was added directly to the mixture. Upon hydration the thread swelled rapidly as individual cell filaments separated from one another. A cover slip was added and the specimens were treated in the standard way. These materials were used to show the detection of a few live cells in a population consisting almost entirely of dead cells.

Fig. 1. Cell viability in macrofibres of strain FJ7 deduced from membrane permeability properties using the Bac Light Bacterial Viability Kit. Each of the first six structures shown were photographed twice using different filters as described in the text. The green images of each structure shown on the left represent viable cells, the red images shown on the right represent dead cells. Panels 1 and 2 show right-handed macrofibres (R). The smallest elements resolved are filament chains of single cells. The single dead cell shown in panel 1 is marked with an arrowhead on the red image. Five dead cells are marked similarly in panel 2. Panels 3, 4, 5 and 6 show left-handed macrofibres (L). Occasional dead cells can be found at various locations in them (arrowheads). Images in all panels except 5 were obtained using a $\times \mathbf{4 0}$ objective. The images in panel 5 were obtained using a $\times 10$ objective to give a greater overview of the fibre structure. Two different samples are shown in panel 7 as controls. The left image of panel 7 was obtained from FJ7 cells grown under conditions where individual cell filaments produce an entangled web rather than a macrofibre. The right image of panel 7 shows cell filaments that were first drawn from a web into a dried bacterial thread then re-hydrated in fresh TB medium. A filter that permits dual excitation of both red and green fluorescence was used to obtain both the images shown in panel 7. The purpose of panel 7 is to illustrate that the fluorescence technique used here is capable of resolving both live and dead cells when either is in the minority in a given cell population. Bars: panels $1-4$ and $6,50 \mu \mathrm{m} ;$ panel $5,200 \mu \mathrm{m}$; panel 7, $20 \mu \mathrm{m}$. 
Cell behaviour in macrofibres
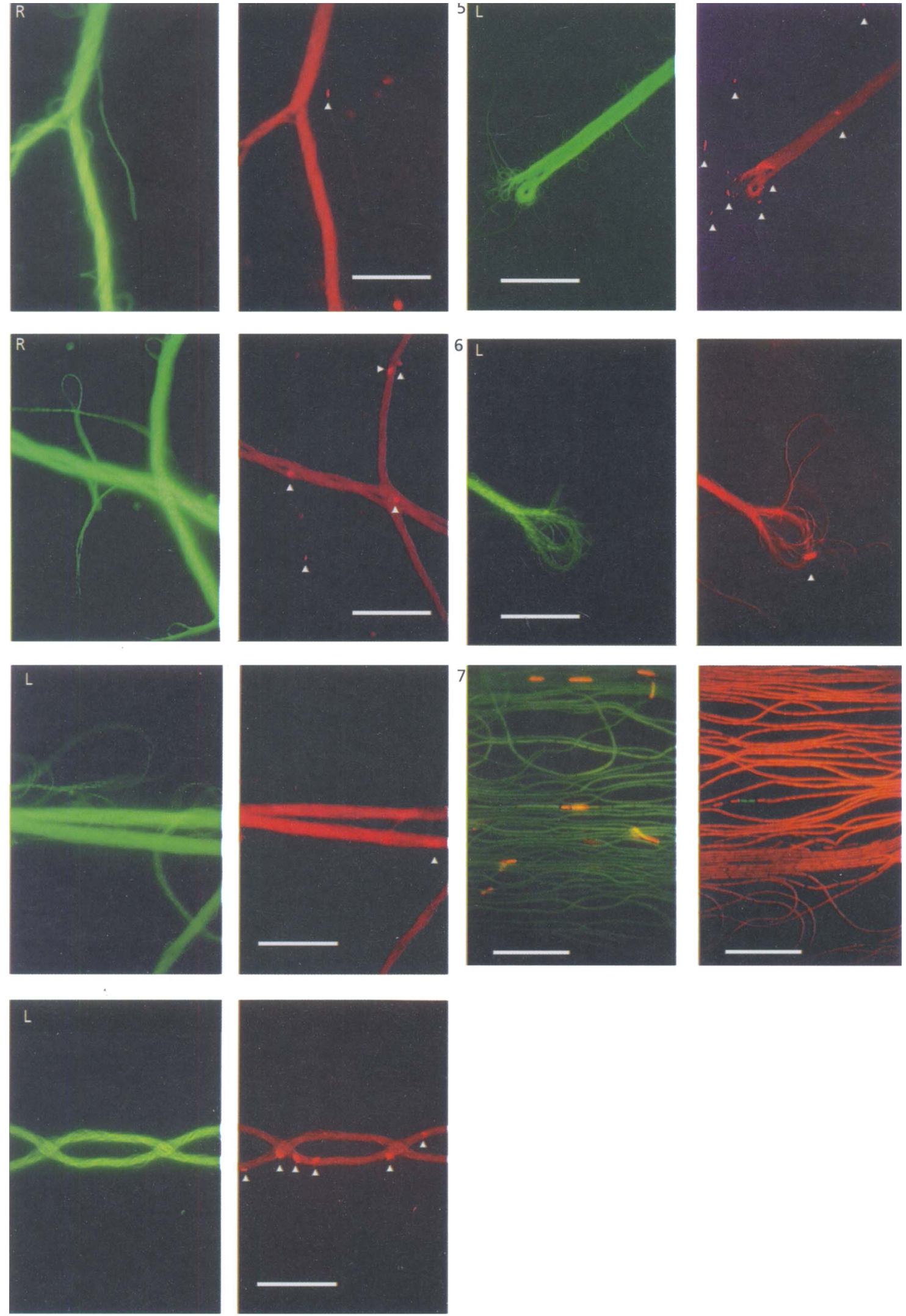

Fig. 1. For legend see facing page. 
(a)
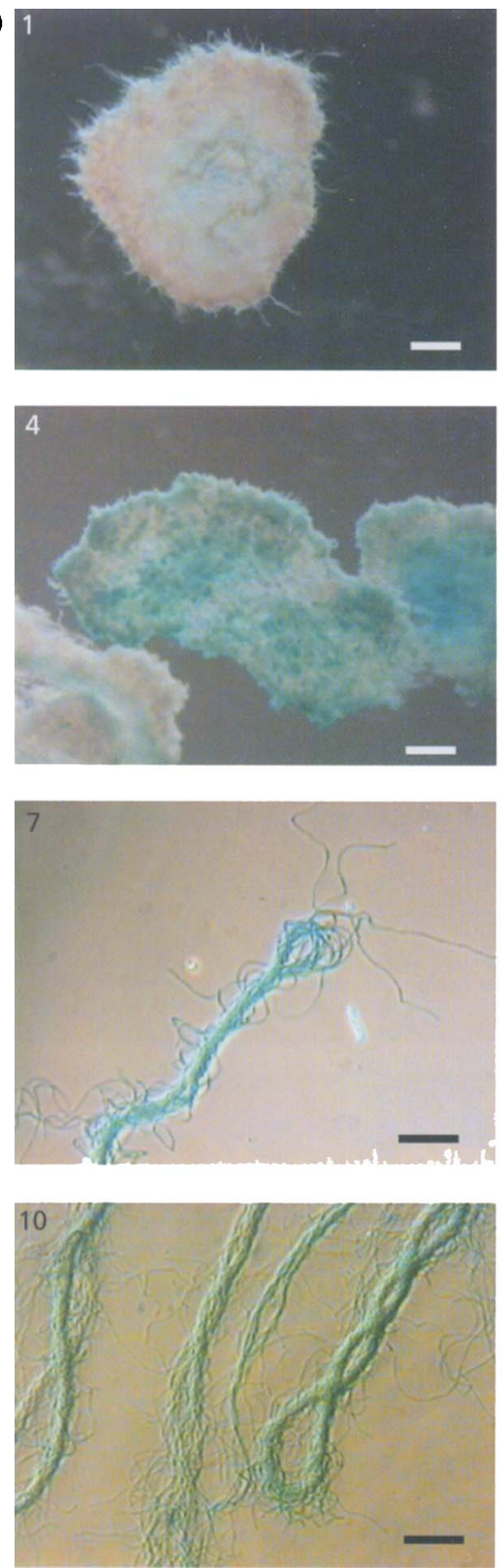
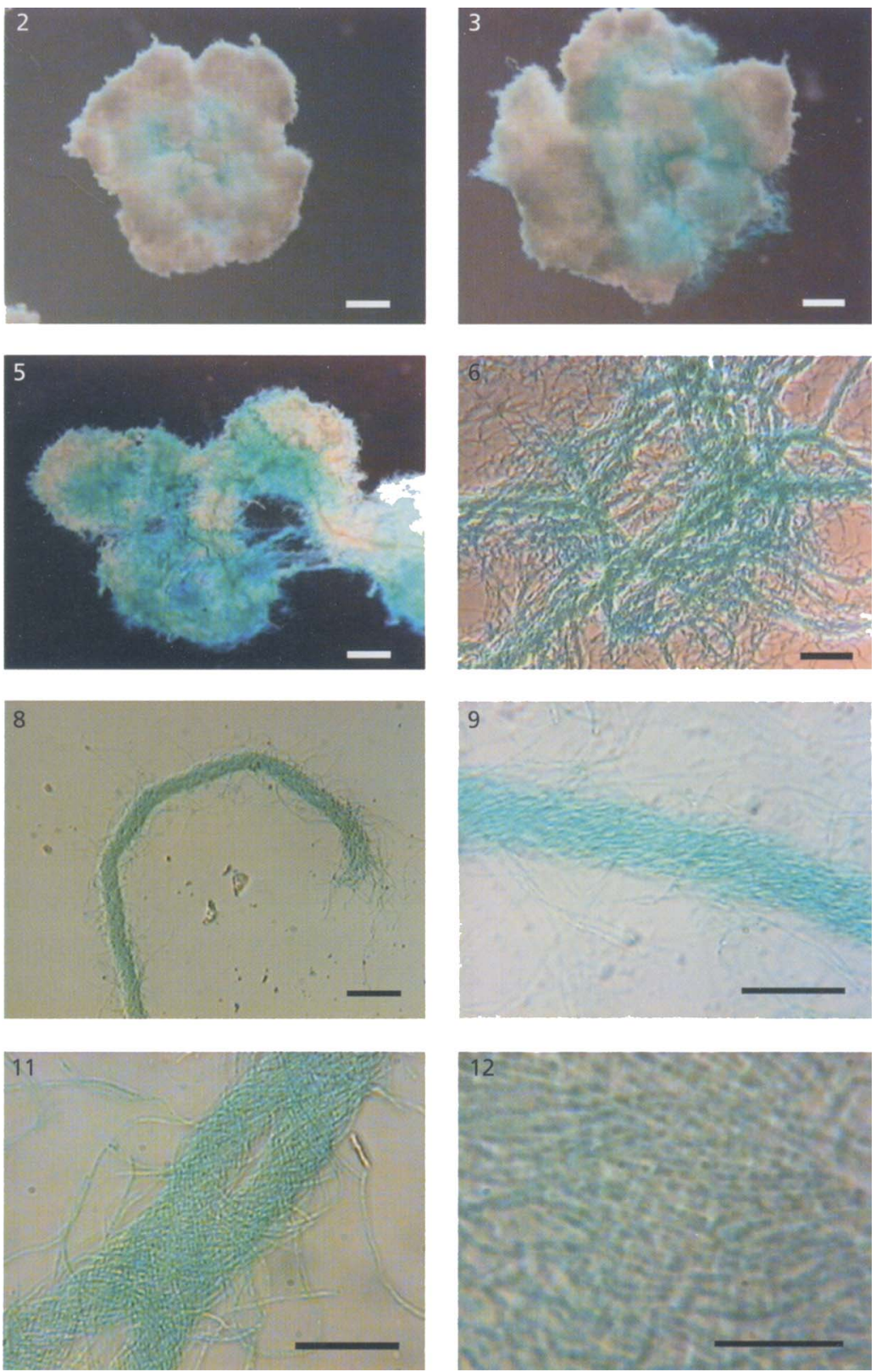

Fig. 2. For legend see facing page.

All specimens were examined using a Zeiss Universal microscope with UV light source and quartz optics. Excitation of green fluorescence (live cells) was obtained using a fluorescence band-pass filter. Red fluorescence (dead cells) was viewed using a rhodamine band-pass filter set. A dual excitation filter (fluorescein/Texas Red) was used to view both red and green fluorescence simultaneously. Images were recorded using Kodak Ektachrome Elite 400 film. Prints were scanned into a computer with a UMAX Vista S-12 scanner (UMAX Technologies) and processed using the Adobe Photoshop program to match by eye the quality of the initial colour slides.

Growth of strain F2b in static fluid media and detection of reporter gene expression. X-Gal was used as the chromogenic substrate to detect $\beta$-galactosidase activity. A $100 \mathrm{mg}$ portion of X-Gal was dissolved in $5 \mathrm{ml} \mathrm{N}, N$-dimethylformamide. This stock was diluted into TB medium to obtain a final X-Gal concentration of $160 \mu \mathrm{g} \mathrm{ml}^{-1}$. Either magnesium sulfate or ammonium sulfate was added to the medium for production of right- or left-handed fibres. The media were inoculated with fragments of a mature macrofibre and grown overnight at $20^{\circ} \mathrm{C}$. The handedness of the helix and degree of twist of the macrofibre structures were determined by light microscopy (Mendelson, 1978). The appearance of blue colour within structures was used as an indication of $\beta$-galactosidase production and reporter gene expression. Macrofibres and their late stage ball-like forms were photographed either directly in situ or at higher magnification from wet mounts using an Olympus X-Tr stereoscopic microscope or a Wild 

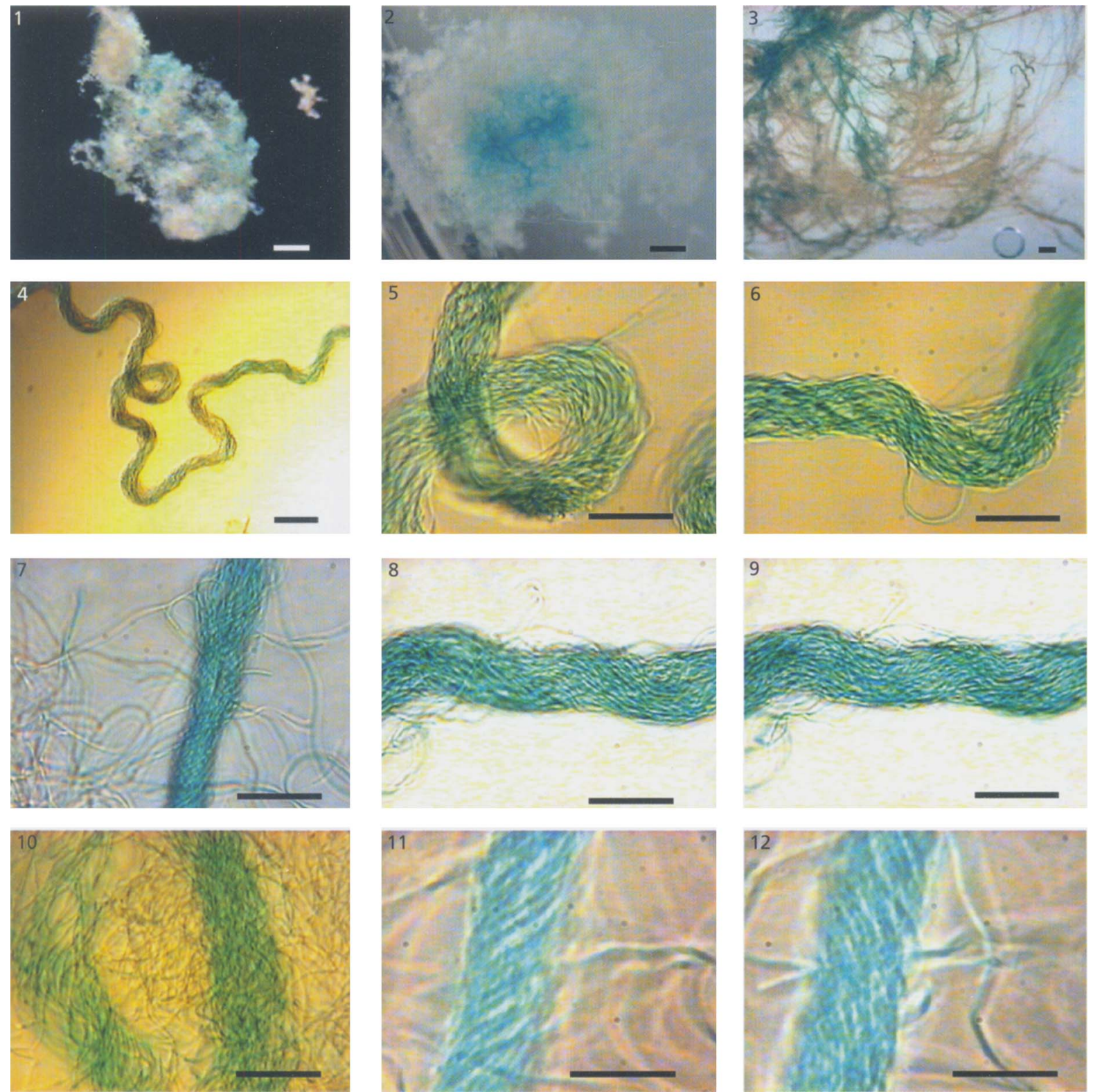

Fig. 2. Initial appearance of reporter gene expression in left (a) and right-handed (b) macrofibres of strain F2b. Fluid cultures $(5 \mathrm{ml})$ were grown at $20^{\circ} \mathrm{C}$ in $60 \mathrm{~mm}$ diameter Petri dishes. Structures were examined daily for initial appearance of blue colour. Images were obtained either in situ at low magnification using a stereoscopic microscope (a, panels 1-5; b, panels 1 and 2) or from wet mounts using a phase-contrast microscope. (a) Panels 10, 11 and 12 show increasing magnification views of the same left-handed structure. Bars: panels $1-5,500 \mu \mathrm{m}$; panels $6-8$ and $10,50 \mu \mathrm{m} ;$ panels 9 and 11, $25 \mu \mathrm{m}$; panel 12, 12.5 $\mu \mathrm{m}$. (b) Panels 4, 5 and 6 show different parts of the same right-handed structure at different magnifications. Panels 8 and 9 show focus on the upper and lower surfaces of the same right-handed structure. Panels 11 and 12 also show images of the upper and lower surfaces, respectively, of a right-handed structure. Bars: panels 1 and 2 , $500 \mu \mathrm{m}$; panels 3 and 4, $50 \mu \mathrm{m}$; panels 5-10, $25 \mu \mathrm{m}$; panels 11 and $12,12.5 \mu \mathrm{m}$.

M20 compound light microscope. Both $35 \mathrm{~mm}$ colour film and a Cohu colour CCD camera were used to obtain images.

\section{RESULTS}

\section{Cell viability in macrofibres}

FJ7 macrofibres produced by overnight growth at $20^{\circ} \mathrm{C}$ in $10 \mathrm{ml}$ cultures of TB medium supplemented with either magnesium or ammonium that had been inoculated with fragments of mature macrofibres grown in the same medium yielded approximately 20 macrofibres per culture. Thus, although the cell density within a fibre is about as high as one can get in a bacterial population, the fibre density in the entire culture was extremely low. The fibre population itself, produced by overnight growth, consisted of individuals at different stages of 
morphogenesis ranging from young fibres with tens or fewer cell filaments in their fibre shaft to older fibres consisting of some hundreds of cell filaments twisted together into their characteristic helical form. Fibres representative of this spectrum were examined using the cell viability staining system.

The helix handedness of all fibres examined was unambiguously determined by observation of the orientation of cellular filaments that lie on the upper and lower surfaces as described previously (Mendelson, 1978: in right-hand fibres, filaments on the upper surface run from lower left to upper right along the length of the fibre axis, while those on the lower surface run from lower right to upper left; the inverse orientations are found in left-handed fibres).

The helix handedness of a macrofibre cannot be determined, however, by examination of micrographs unless the focal plane of the image is known and coincides with either the upper or lower surface of the structure. The micrograph of a fibre that lies out of plane may contain regions in which focus is on the upper surface, the lower surface or in between (see Mendelson et al., 1997 for an example of a through focus series). In the data shown here it was sometimes necessary to purposely focus at some intermediate level to illustrate a particular cell type (usually the dead cell). Helix orientation cannot be discerned from such images. The helix handedness of these specimens was, however, always determined independently by through focus. As expected, all fibres from any given culture were always of the same handedness. Approximately 20 fibres of each hand were examined in detail with respect to red/green fluorescence after staining with the cell viability reagents. Representative examples of left- and righthanded structures are shown in Fig. 1.

A comparison of the green and red fluorescent images in Fig. 1 shows that virtually all cells in fibres of either helix hand are viable. Because of the intensity of fluorescence, greater details of the cellular structure can usually be resolved in the green images. The limits of individual cells are evident and individual filaments within bundles can be discerned. Single cell filaments, double-stranded helical forms and higher order structures are all present. So too are terminal loops and fibre shafts with filament bundles at various stages of amalgamation. In some cases where a single filament lies separated from others it is possible to distinguish a dead cell from the remainder solely on the basis of missing green fluorescence [see Fig. 1, panel 1, for an example]. Within bundles and larger structures, however, the red fluorescent images must be examined to detect inviable cells. When viewed directly in the microscope the red fluorescence difference between background observed in viable cells and a positive response in dead cells is striking. The photographic images shown do not do justice to the actual difference. Nevertheless, even when dead cells are found within a large fibre shaft with proportionally greater background red fluorescence, the true positives are easily identified. Moreover, a perusal of the images in Fig. 1 shows that dead cells can be located at different places in fibres. There does not appear to be any particular region therefore where cells die preferentially.

In view of the low frequency of red-fluorescence-positive dead cells found in macrofibres, we examined controls consisting of FJ7 single cell filaments obtained from web cultures and filaments derived from hydrated bacterial threads that are known to contain predominantly live and dead cells, respectively. The images, shown in Fig. 1, panel 7, were obtained using a dual excitation UV light filter that enables both red and green fluorescent cells to be displayed simultaneously. (Red and green fluorescence induced by the dual excitation filter was previously shown to correspond to red and green fluorescence induced with the individual single excitation filters that were used to obtain the other images shown in Fig. 1.) The results indicate that a small minority of live FJ7 cells in a population of dead cells (the image shown on the right of panel 7) as well as a small minority of dead cells in a population of live cells (the image shown on the left of panel 7) can be resolved by the live/dead staining reaction.

\section{Reporter gene expression in macrofibres}

Reporter gene expression was examined during formation of $B$. subtilis macrofibres at $20^{\circ} \mathrm{C}$ in media containing X-Gal. The young fibres produced following subculture from mature fibres were examined for the initial appearance of blue colour. In both left- and righthanded forms (Fig. 2a and b, respectively), colour arose initially at two locations: (i) in large fibres that were embedded in ball-like structures and (ii) in smaller fibres situated on the outer surface of the ball structures. The large fibres that became positive early during fibre morphogenesis appear to be the core structures from which the ball-like forms developed, whereas the smaller peripheral fibres appear to have arisen later in development by surface outgrowths from the older core. Blue colour spread throughout the structures from these initial foci as the structures became older. Individual fibres were also present in older cultures along with the prevalent ball-like forms. Some of them were uniformly blue throughout their structure. Others had regions lacking blueness although a majority of their cells were blue. Clearly the level of reporter gene enzyme activity varied as a function of space and time in F2b macrofibres.

When old F2b structures that had become totally blue were transferred from their original cultures to fresh medium of the same composition, new growth arose primarily from the surface of each structure. The new cells produced in these regions were always white. An example is shown in Fig. 3. In this particular structure a surface fibre attached to the basic ball form at both its ends developed three foci of new growth (the white regions in Fig. 3, panel b), each of which was separated from the others by a segment of the original fibre that remained blue. With continued incubation, however, all 

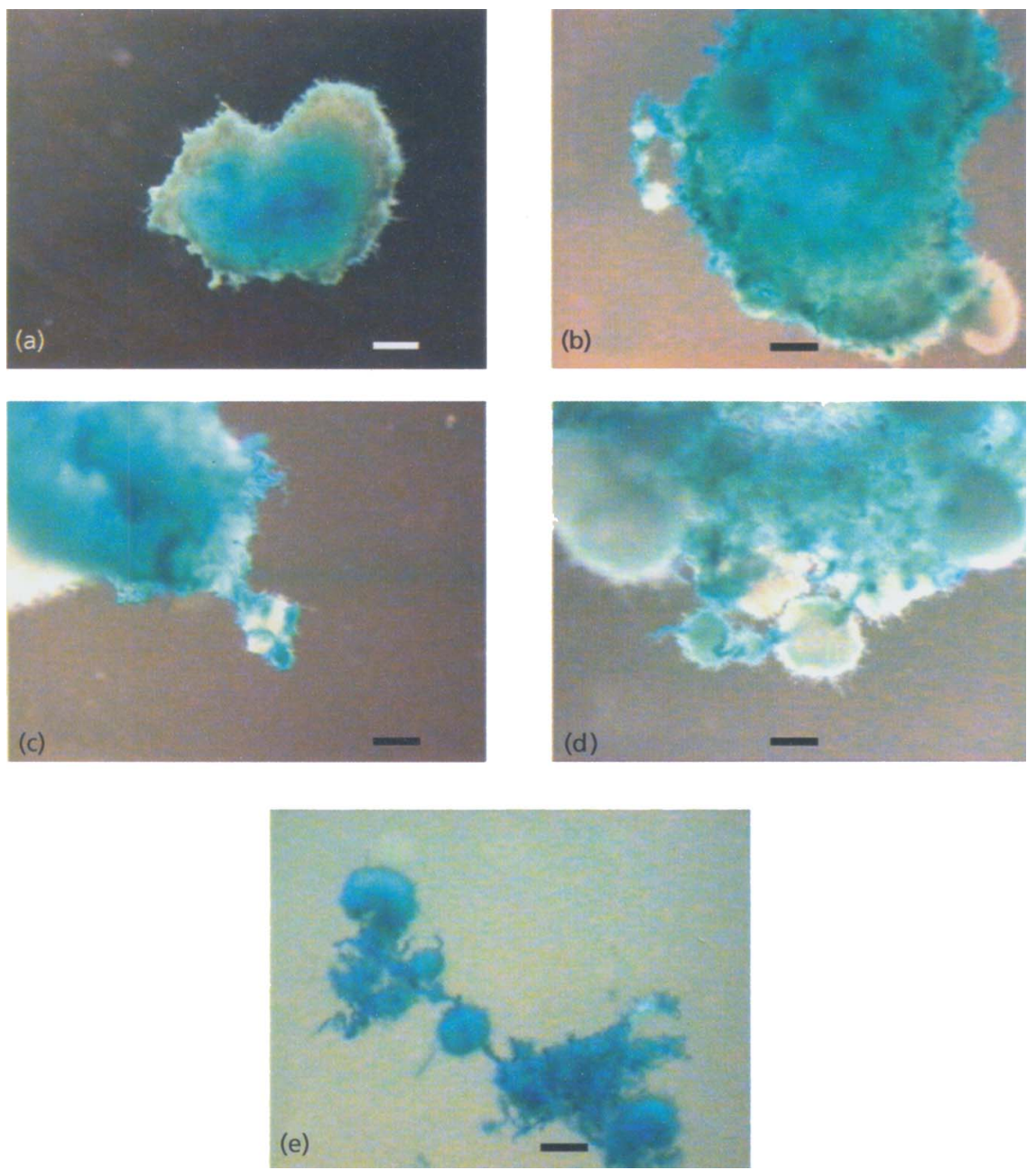

Fig. 3. Gene expression patterns produced following subculture of a mature F2b structure showing heavy reporter gene expression to fresh medium of the same composition. The initial structure shown in (a) was produced by $5 \mathrm{~d}$ growth of a left-handed fibre at $20^{\circ} \mathrm{C}$ in TB medium containing ammonium sulfate and X-Gal. After being photographed the structure was transferred to fresh medium. (b), (c), (d) and (e) show the same structure 2, 3, 4 and 8 d, respectively, after transfer. Images were obtained using an Olympus X-Tr stereoscopic microscope. The peripheral blue fibre within which three new regions of growth appeared (white regions lacking expression) located on the left of the image in (b) can be identified in (c), (d) and (e). All three regions continued to grow and eventually expressed the reporter gene. The largest of the three regions is located in the centre of the structure shown in (e). Bars: (a), $500 \mu \mathrm{m}$; (b), (c) and (e), $320 \mu \mathrm{m} ;(\mathrm{d})$, $200 \mu \mathrm{m}$.

of the newly formed white structures became blue (Fig. 3 , panel e). This pattern - an old blue structure upon subculture yields new growth that is white, new white structure eventually becomes blue as it ages - has been seen in many parallel experiments. In F $2 b$ structures there is a distinct temporal appearance of the reporter gene product.

\section{DISCUSSION}

Little is known about the behaviour of individual bacterial cells that are part of multicellular entities in comparison to their behaviour when grown as individual cells in conventional fluid cultures (Shapiro, 1997; Kroos \& Kaiser, 1987; Belas et al., 1986). Bacterial macrofibres provide a convenient means to examine such differences. Macrofibres are easily produced, well characterized in terms of their structural organization and morphogenesis, and the strains that produce them can be grown as either single cell filaments, conventional colonies or as macrofibres. A typical macrofibre of $1 \mathrm{~mm}$ length consists of a single cell filament, approximately $10 \mathrm{~cm}$ in length, containing about $2.5 \times 10^{4}$ cells linked to one another in tandem. The cell filament in a macrofibre is twisted and folded repeatedly upon itself, thereby producing parallel stretches of the filament that form the shaft of the fibre and loops at both ends of the shaft. Structures resembling the early stages of macrofibre formation have been found in nature, suggesting that the macrofibre multicellular form is not simply a laboratory 
curiosity (Roberts, 1938). The macrofibre-producing strains used in the studies described here have been characterized previously in terms of their macrofibre behaviour, and also as conventional colonies grown on agar surfaces. The properties of individual cells within macrofibres have not been examined previously, however.

In this study we show for the first time that virtually all the cells in macrofibres are viable. Other multicellular bacterial forms such as colonies and biofilm populations often contain many dead cells (Yarmolinsky, 1995; Roszak \& Colwell, 1987). Although macrofibre structure imposes significant constraints on its cells, such as extreme shape deformation of the cells located in terminal loops and shielding of the cells trapped in the interior of a fibre from direct access to the growth medium, the cytochemical live/dead detection system that we used reveals that very few dead cells are present in any given macrofibre. The covalent linkage of cells into a single filament in macrofibres prevents the use of culturing methods to independently confirm the ability of every cell in a macrofibre to grow. Nevertheless, the current finding, coupled with earlier observations showing that macrofibre growth rates are comparable to those of single cells grown in the same environment (Mendelson, 1992), that cell ultra-structure appears normal in macrofibre cells (Mendelson, 1982, and unpublished work of R.M. Cole) and the very uniform structure of double-helical forms that arise during the early stages of macrofibre production (Mendelson, 1976) strongly suggest that constraints imposed by macrofibre multicellularity do not cause lethality. The random locations of occasional dead cells in macrofibres supports this contention.

We also describe in this communication initial studies of gene expression in macrofibre cells. Although the system we used is far from ideal (neither the host promoter controlling the reporter gene nor the $\sigma$ factor regulating the host promoter are known at present and the X-Gal assay as used is not a direct quantitative measure of gene expression), the results obtained show clearly that not all cells in F2b macrofibres behave in the same manner. There are both spatial and temporal differences in the degree of $\beta$-galactosidase activity in macrofibre structures, suggesting differential gene expression during morphogenesis. The pattern observed in colonies of $\mathrm{F} 2 \mathrm{~b}$ grown on media of the same composition solidified with agar is different. During colony morphogenesis activity appears early, is uniformly distributed and is judged to be high on the basis of blue colour intensity (Salhi \& Mendelson, 1993). In contrast, $\beta$-galactosidase activity in F2b macrofibres appears first at initial foci, then spreads to neighbouring regions. This, plus the fact that fibres produced late in such cultures show uniform activity throughout, while new growth arising out of old structures upon subculture do not have activity, suggests that the cells in macrofibres are responsive to local as well as global conditions of the environment.

The demonstration that nearly all cells in macrofibres are viable and that gene expression can be resolved at the individual cell level in them shows that it is feasible to use macrofibres to examine developmental regulatory processes in prokaryotes similar to those usually studied in eukaryotic systems (Lawrence, 1992). By doing so we hope to learn how individual cell behaviour governs in a hierarchical way that of the higher order multicellular state (Mendelson et al., 1997).

\section{ACKNOWLEDGEMENTS}

We thank S.D. Whitworth for technical assistance. This research was supported by a grant from the National Center for Research Resources to N.H.M. K.E.S. was supported by the University of Arizona Undergraduate Biology Research Program.

\section{REFERENCES}

Belas, R., Simon, M. \& Silverman, M. (1986). Regulation of lateral flagella gene transcription in Vibrio parahaemoliyticus. J Bacteriol 167, 210-218.

Ben-Jacob, E., Schochet, O., Tenenbaum, A., Cohen, I., Cszirok, A. \& Vicesek, T. (1994). Generic modeling of cooperative growth patterns in bacterial colonies. Nature 368, 46-49.

Kroos, L. \& Kaiser, D. (1987). Expression of many developmentally regulated genes in Myxococcus depends on a sequence of cell interactions. Genes Dev 1, 840-854.

Lawrence, P. A. (1992). The Making of a Fly. London: Blackwell.

Mendelson, N. H. (1976). Helical growth of Bacillus subtilis: a new model of cell growth. Proc Natl Acad Sci USA 73, 1740-1744.

Mendelson, N. H. (1978). Helical Bacillus subtilis macrofibers: morphogenesis of a bacterial multicellular macroorganism. Proc Natl Acad Sci USA 75, 2478-2482.

Mendelson, N. H. (1982). Dynamics of Bacillus subtilis helical macrofiber morphogenesis: writhing, folding, close packing and contraction. J Bacteriol 151, 438-449.

Mendelson, N. H. (1992). Self-assembly of bacterial macrofibers: a system based upon hierarchies of helices. Mat Res Soc Symp Proc 255, 43-54.

Mendelson, N. H. \& Favre, D. (1987). Regulation of Bacillus subtilis macrofiber twist development by ions: effects of magnesium and ammonium. $J$ Bacteriol 169, 519-525.

Mendelson, N. H. \& Salhi, B. (1996). Patterns of reporter gene expression in the phase diagram of Bacillus subtilis colony forms. $J$ Bacteriol 178, 1980-1989.

Mendelson, N. H. \& Thwaites, J. J. (1989). Do forces and the physical nature of cellular materials govern biological processes? Comment Theor Biol 1, 217-235.

Mendelson, N. H., Salhi, B. \& Li, C. (1997). Physical and genetic consequences of multicellularity in Bacillus subtilis. In Bacteria as Multicellular Organisms, pp. 339-365. Edited by J. A. Shapiro \& M. Dworkin. New York: Oxford University Press.

Roberts, J. L. (1938). Evidence of a rotational growth factor in Bacillus mycoides. Science 87, 260-261.

Roszak, D. B. \& Colwell, R. R. (1987). Survival strategies of bacteria in natural environments. Microbiol Rev 51, 365-379.

Salhi, B. \& Mendelson, N. H. (1993). Patterns of gene expression in Bacillus subtilis colonies. J Bacteriol 175, 5000-5008.

Shapiro, J. A. (1997). Multicellularity: the rule, not the exception. Lessons from Escherichia coli colonies. In Bacteria as Multicellular Organisms, pp. 14-49. Edited by J. A. Shapiro \& M. Dworkin. New York: Oxford University Press. 
Thwaites, J. J. \& Mendelson, N. H. (1985). Biomechanics of bacterial walls: studies of bacterial thread made from Bacillus subtilis. Proc Natl Acad Sci USA 82, 2163-2167.

Yarmolinsky, M. B. (1995). Programmed cell death in bacterial populations. Science 267, 836-837.

Youngman, P. (1987). Plasmid vectors for recovering and exploit- ing Tn917 transpositions in Bacillus and other Gram-positive bacteria. In Plasmids, A Practical Approach, pp. 79-103. Edited by K. G. Hardy. Oxford: IRL Press.

Received 11 April 1997; revised 21 July 1997; accepted 4 August 1997. 